\title{
PATHOGENIC MECHANISMS OF GENERALIZED PERIODONTITIS FROM THE POSITION OF POLYMORPHISM OF NUCLEAR TRANSCRIPTION FACTOR NF-KB1
}

\author{
N. V. Hasiuk ${ }^{1}$, L. E. Vesnina2, O. A. Shlykova², O. V. Izmailova² \\ ${ }^{1}$ I. HORBACHEVSKY TERNOPIL STATE MEDICAL UNIVERSITY, TERNOPIL, UKRAINE \\ ¿UKRAINIAN MEDICAL DENTAL ACADEMY, POLTAVA, UKRAINE
}

Background. Periodontal diseases are a topical issue of contemporary dentistry because they are accompanied by severe morphological and functional disorders of maxillodental system; and are characterized by polyetiology and a number of metabolic disorders.

The purpose of this study was to substantiate the pathogenic mechanisms of generalized periodontitis in relation to polymorphism of nuclear transcription factor NF- $K B 1$.

Objective. The aim of the study was to determine the genetic factors in the development of generalized periodontitis and the relationship of this parameter with immunohistochemical affiliation for cellular infiltrate of the lamina propria of gum at this nosology in young people. Hence, 2 groups were formed: I - control and II - observational.

Methods. Polymorphic gene section NF-KB1 was determined using the cells of buccal epithelium of the examined people by means of polymerase chain reaction. Collection of material was performed with sterile disposable dental brush, followed by the introduction of a reagent in ependorph with DNA Express reagent (LyTeh NPF, Russia). Genome deoxyribonucleic acid was isolated by DNA Express set (LyTeh, Moscow).

Results. The lack of correlation in this case indicates that no matter how parameters change, relatively major genotype (Del/Del) in this case is unchanged and the determining factor causes the development of generalized periodontitis, clinical picture of which is rapidly progressing.

Conclusions. Results of correlation analysis proved that genotype (Del/Del), as polymorphic variant of gene transcription factor NF-KB1, was significantly associated with the emergence of rapidly progressive periodontitis in young people.

KEY WORDS: gingival; generalized periodontitis; cells; nucleus; transcription factor.

\section{Introduction}

Periodontal diseases are an urgent matter of contemporary dentistry because they are accompanied by severe morphological and functional disorders of maxillodental system; and are characterized by polyetiology and a number of metabolic disorders [1, 7, 12].

In case of inflammatory diseases of periodontal tissues, which are regarded today as polyetiological, such factors as genetic and non-genetic influence on each other with the effects that are not always clearly defined [10, 13]. In most cases, both types of factors influence the pathogenesis and severity of clinical symptoms.

Corresponding author: Natalya Hasiuk, Department of Therapeutic Dentistry, I. Horbachevsky Ternopil State Medical University, 1 Maidan Voli, Ternopil, Ukraine, 46001 Phone number: +380352259802

E-mail: gasyknv@tdmu.edu.ua
There is various systematics as well as clinical and morphological forms of generalized periodontitis [11] depending on aetiology, course of severity and immune response [14]. Polymorphic clinical features of this nosology are associated with different composition of subgingival microflora. The factors that affect antimicrobial response and new metabolic status of the organism are also important [6].

The problem of early diagnosis of generalized periodontitis, the development of effective measures of its prevention and treatment that is aimed at achievement of positive longterm results are topical matter of contemporary dentistry $[2,3]$.

It is established that various exogenous and endogenous factors and triggers [4] contribute to development of generalized periodontitis, but the influence of these factors is studied insufficiently [9]. 
Also, the established ideas and concepts [5] do not explain fully the existing differences in the identification of risk factors and progression of generalized periodontitis, its course, ambiguous outcomes under the same conditions. Due to the usual difference in the rate of generalized periodontitis progression in patients treated with the standard schemes, the biological heterogeneity of the disease and its possible complex causes are suggested.

In this regard, it would allow predicting the clinical course of generalized periodontitis in patients with this nosology. Therefore, studying the relationship of this disease with genetically determined factors should be recognized as perspective.

The purpose of this study was to substantiate the pathogenic mechanisms of generalized periodontitis from the position of polymorphism of nuclear transcription factor NF-kB1 that is based on patterns that determine the relationship between the genetic profile of an individual and the nature of cellular infiltrate of the lamina propria of gums in patients with generalized periodontitis.

\section{Methods}

To determine the genetic factors of generalized periodontitis development and the relationship of this parameter with immunohistochemical affiliation for cellular infiltrate the lamina propria of gum of this nosology in young people, 2 groups for observation were formed: I (control - 45 people with intact periodontium) and II (55 people - patients with generalized periodontitis).

Dental examination of 100 people was conducted in accordance with the standards of diagnosis and treatment of dental patients (the decrees of the Ministry of Health of Ukraine "On approving of the protocols of medical care in specialties "orthopedic dentistry", "therapeutic dentistry", "surgical dentistry", "orthodontics", "pediatric dentistry", "pediatric surgical dentistry" from 28.12.2002 №507 and "On approval of standards of medical care and quality of medical care" from 28.12.2002 №507).

Determination of polymorphic gene section NF-KB1 was performed using the cells of buccal epithelium of the examined people by means of polymerase chain reaction. Collection of material was performed with sterile disposable dental brush followed by the introduction of a reagent in ependorph with DNA Express reagent (LyTeh NPF, Russia). Genome deoxyribonucleic acid was isolated by DNA Express set (LyTeh, Moscow).
Polymorphic gene area NF-kB1 (rs28362491) was amplified by polymerase chain reaction. The final volume of the reaction mixture was $25 \mathrm{mkl}$ and contained:

- specific oligonucleotide primers - $66 \mathrm{ng}$ each,

direct - 5'-TGGGCACAAGTCGTTTATGA-3';

reverse - 5'-CTCGAGCCGGTAGGGAAG-3';

- 2,5 mkl 10 times buffering for amplification;

- $2 \mathrm{mM}$ magnesium chloride;

- 0,2 mM deoxy nucleotide triphosphate (dNTP);

- 2,5 units. Taq DNA polymerase;

- 20-50 ng of genomic DNA.

In the tube containing these material $25 \mathrm{mg}$ of mineral oil was placed.

Amplification was performed by means of Tertsyk thermocyclers (DNA Technology Ltd, Russia).

To identify alleles, the restriction analysis of amplicons using restriction enzyme PfIMI (SybEnzym, Russia) at $37^{\circ} \mathrm{C}$ was performed.

Products cleavage of polymorphic gene section NF-kB1 was detected by horizontal electrophoresis in $2 \%$ agarose gel in a single TBE (50 mM Tris- $\mathrm{H}_{3} \mathrm{BO}_{3}$ and $2 \mathrm{mM}$ EDTA, $\mathrm{pH} 8,0$ ), for 2 hours at a voltage of $2 \mathrm{~V}$ per $1 \mathrm{~cm}$ of gel. pBR322/Alu I was used as a molecular weight marker of DNA. Gels were stained by etydiumom bromide followed by visualization of the results in the UV light.

According to the research objectives of the process of immunohistochemical studies, monoclonal antibodies CD-3 (clone SP7, DakoCytomation), CD-4 (clone 4B12, DakoCytomation), CD-20 (clone L26, DakoCytomation) wereused. Theresults of of immunohistochemical studies visualization were carried out by En Vision system (DakoCytomation). The degree of expression of markers was determined by the colour intensity of specific patterns in brown colour. In some cases for structure differentiation, the obtained sections were stained additionally with Maher's hematoxylin. The degree of expression was evaluated individually for each marker and determined on at least (8-10) randomly selected fields of Biorex-3 BM-500T digital light microscope.

The results of clinical and complex morphological studies were analysed using a number of biostatistics methods. Statistical research was performed at the Department of Statistical Research of SIHE "I. Horbachevsky Ternopil State Medical University of the Ministry of Health of Ukraine". The data was processed by the licensed program Statistica (StatSoft). 
To analyse the relationship of parameters that were studied (immunohistochemical profile of cellular infiltrate of the lamina propria of gums) the correlation coefficient ( $r$ )Spearman was determined. The correlation coefficient was considered statistically significant at $p<0.05$ [8].

The value of the correlation coefficient represents the degree of closeness between the values to a linear function, which correspond to the values \pm 1 correlation coefficient. If $r x y>0$, the correlation is positive; it means that with increasing of one value, a second value increases in average. If $r x y<0$ correlation is negative, that is with increasing of one value, a second on average is decreased. If there is no statistical relationship between the values, the correlation coefficient is zero. The level of p-test (statistical significance) depends on the value of the correlation coefficient and the size of the experimental group for which the determination of the correlation coefficient is made. In the statistical processing of results, molecular genetic studies, in accordance with certain genotype (Del/Del), (Del/Ins), (Ins/Ins), as a basis for comprehension of the genetic structure of the population the law of genetic equilibrium by Hardy-Weinberg was applied. Due to this law, according to data on the frequency of recessive phenotype in the population that have homozygous genotype, the prevalence of polymorphic variants of NF-kB1 was calculated in the exemined groups. Statistical justification of probability differences of genotypes distribution was performed using $\chi^{2}$ test adjusted for continuity by Yates [8].

\section{Results}

The molecular genetic study included a selection of polymorphic gene sites of nuclear transcription factor NF-kB1 cells of buccal epithelium by polymerase chain reaction.

The specificity of immunohistochemical markers expression on the plasmolemma of cells that formed inflammatory infiltrates made it possible to distribute them to the group and allocate three immunohistochemical profiles of infiltrates.

First immunohistochemical profile was characterized by prevalence of $\mathrm{T}$ lymphocytes infiltrates, which were expressed on plasmolemma of immunohistochemical marker CD-3 multi-protein complex that was express on the surface of T-lymphocytes. The increased level of the marker expression on cells' plasmolemma, which appeared in a dark brown colour, was determined (Fig. 1).
Stated immunohistochemical profile correlated with the frequency of exacerbations 3 times a year, creating a statistically significant relationship with correlation coefficient $(r=0.58$, in $p<0.05$ ).

Second immunohistochemical profile was characterized by a predominance of T-lymphocytes in infiltrates that wereexpressed on plasmolemma by immunohistochemical marker CD-4 that was one of the proteins cluster of differentiation, monomeric transmembrane glycoprotein of superfamily Ig with a molecular weight of $55 \mathrm{kDa}$, which was the marker of T-helper cells.

In our research, a high level of the marker expression on the plasmolemma of cells, which appeared in a dark brown colour, was proved (Fig. 2).

The stated immunohistochemical profile correlated with the frequency of exacerbations once a year, creating a statistically significant

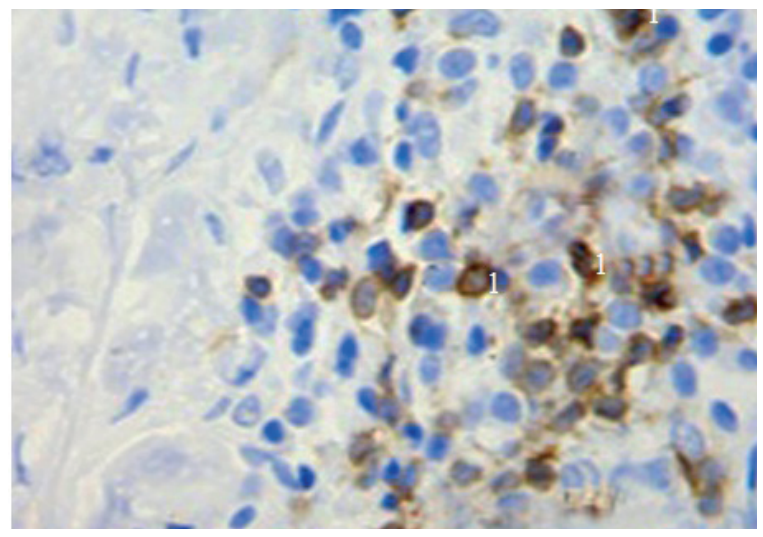

Fig. 1. The expression of CD-3 marker in the complex of cellular infiltrates in the lamina propria of gums in the course of generalized periodontitis. Immunohistochemical method, extra Meyer hematoxylin staining. Magnification: x 1000. 1 - high degree of expression.

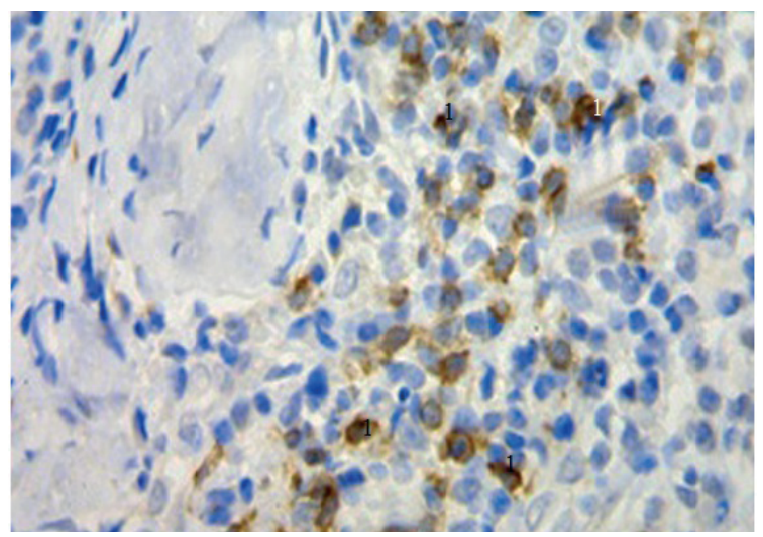

Fig. 2. The expression of CD-4 marker in the complex of cellular infiltrates in the lamina propria of gums in the course of generalized periodontitis. Immunohistochemical method, extra Meyer hematoxylin staining. Magnification: x 1000. 1 - high degree of expression. 
relationship with correlation coefficient $(r=0.51$, in $p<0.05$ ).

Third immunohistochemical profile was characterized by a predominance of cells infiltrates that were expressed in plasmolemma by immunohistochemical marker CD-20 or B-lymphocyte antigen - a protein, co-receptor located on the surface of B-lymphocytes. It was established that in cell infiltrates of the lamina propria, high and moderate expression was determined in a dark brown and light brown coloration of cells (Fig. 3).

The stated immunohistochemical profile correlated between frequency of exacerbations 5 times a year, creating a statistically significant relationship, and correlation coefficient $(r=0.94$, at $p<0.05)$. The correlation analysis of clinical dental and immunohistochemical research with determination of correlation coefficient $(r)$ Spearman confirmed the correlation between frequency of exacerbations and immunohistochemical profile of an individual. The maximum value of the correlation coefficient $(r=0.91)$ has CD-20 immunohistochemical profile and probable frequency of exacerbations 5 times a year. The determined correlation between CD-3 immunohistochemical profile and frequency of exacerbations - 3 times per year $(r=0.58)$ and correlation between CD-4 immunohistochemical profile and frequency of exacerbations once per year $(r=0.51)$.

\section{Discussion}

The stated presence of correlations between anamnesis, clinical indicators and character of CD- belonging to cellular infiltrate of the lamina propria of the gums in generalized periodontitis makes it possible to systematize generalized

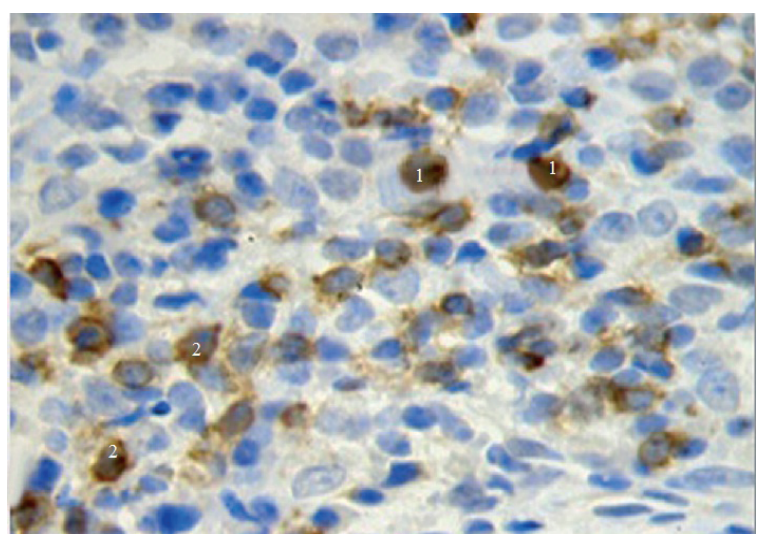

Fig. 3. The expression of CD-20 marker in the complex celIular infiltrates of the lamina propria of the gums during GP. Immunohistochemical method, extra Meyer hematoxylin staining. Magnification: x 1000. 1 - high degree of expression; 2 - moderate degree of expression. periodontitis in young people on clinical and morphological forms depending on the predominance of inflammatory infiltrates of $T$ or $B$ cells. The intensity of the course of inflammatory and destructive processes in periodontium is determined by reorganization of specific local immunity, which is provided by cellular and humoral components, which is reflected in the change of immunohistochemical profile of inflammatory infiltrates. The obtained results of correlation analysis enable to systematize generalized periodontitis relatively, in the examined people, on 'stable' with exacerbation rate once a year and CD-4 immunohistochemical profile; 'conditionally stable', with frequency of exacerbations 2-3 times a year and CD-3 immunohistochemical profile; 'progressive', with frequency of exacerbations 3-5 times a year and CD-20 immunohistochemical profile. Therefore, due to immunohistochemical features of cellular infiltrate of the lamina propria of gums in the course of generalized periodontitis it becomes possible to predict clinical course of nosology.

The detailed analysis of molecular genetic studies proved that $30(55 \%)$ patients with generalized periodontitis had genotype (Del/ Ins) - heterozygote, another 16 people (29\%) genotype (Ins/Ins) - homozygote, sized fragment of polymorphic area $94 \mathrm{bp}$. The most pronounced clinical manifestations of inflammatory and degenerative changes in periodontal tissues, characterised by clinical manifestation of rapidly-progressing periodontitis, was observed in 9 persons (16\%) with genotype (Del/ Del) - homozygote.

The results of correlation analysis, with definition of Spearman correlation coefficient $(p<0.05)$, provides the data on the lack of correlation between the defined parameters in patients with polymorphic variant (Del/Del). The lack of correlation in this case indicates that despite the parameters changes (PMA, available or no concomitant somatic pathology, etc.), relatively major, in this case genotype (Del/Del) is unchanged and the determining factor causes the development of generalized periodontitis, clinical picture of which is to rapidlyprogressing.

At the same time, in 30 people with genotype (Del/Ins) the direct correlation by Spearman $(p<0.05)$ was identified between the immunohistochemical profile of an individual and available concomitant somatic pathology with correlation coefficient $(r=0.60)$ between bad habit (smoking) and PMA index ( $r=0.17)$, which 
indicates a direct role of these parameters in the emergence and development of generalized periodontitis.

It should be noted, the significant inverse correlation by Spearman $(p<0.05)$ between PMA index and concomitant somatic pathology $(r=-0.40)$ and immunohistochemical profile of an individual $(r=-0.37)$ was evidenced.

The characteristic features of the defined correlation connections between parameters makes it possible to state that the registered clinical manifestations of generalized periodontitis in case of this polymorphic variants are not genetically determined, but possibly are caused by exogenous pathogens and local factors $[12,13]$ that initiate the development of induced generalized periodontitis, considering possible ways of activating transcription factor NF-kB1, which contributes to the emergence or enhances pathological responses in periodontal tissues (Table 1).

The absence of significant direct correlation by Spearman $(p<0.05)$ between genotype parameters (Ins/Ins) should be noted; this leads to the assumption that the pathogenic mechanisms of generalized periodontitis in patients with this variant have polymorphic genetic component, but the features of clinical manifestations are more favourable and are characterized by chronic long course in comparison to the polymorphic variant (Del/Del). The results of correlation analysis provide an opportunity to offer systematics of generalized periodontitis due to the nature of inflammatory infiltrates of $\mathrm{T}$ or $\mathrm{B}$ cells and genotype (Table 2).

The control group consisted of patients with intact periodontal genotype (Del/Del): there were 5 people (11\%); so the risk group for morbidity of generalized periodontitis have been developed and was recommend taking these individuals under medical supervision. 23 people $(51 \%)$ with polymorphic variant of transcription factor NF-kB1 (Del/Ins) constituted a risk group with bad habits and concomitant somatic pathology that in the future would create preconditions for development of induced generalized periodontitis on the background of vascular disorders of the lamina propria of gums. 17 people (33\%) with polymorphic variant of the transcription factor NF-kB1 (Ins/Ins) constituted a risk group in the case of impact of local adverse factors and current tooth-jaw anomalies and deformities (see Table 1).

Table 1. Distribution of polymorphic variants of gene transcription factor NF-KB1 in patients with intact periodontium of tissues and with generalized periodontitis

\begin{tabular}{|l|c|c|c|}
\hline \multicolumn{1}{|c|}{ Characteristics of examined } & $\begin{array}{c}\text { Homozygotes } \\
(\mathrm{D} / \mathrm{D})\end{array}$ & $\begin{array}{c}\text { Heterozygotes } \\
(\mathrm{D} / \mathrm{I})\end{array}$ & $\begin{array}{c}\text { Homozygotes } \\
(\mathrm{I} / \mathrm{I})\end{array}$ \\
\hline $\begin{array}{l}\text { Patients with intact periodontium } \\
\text { (45 persons) }\end{array}$ & $\begin{array}{c}23 \\
(11 \%)\end{array}$ & $(51 \%)$ & 17 \\
\hline $\begin{array}{l}\text { Patients with generalized periodontitis } \\
\text { (55 persons) }\end{array}$ & 9 & 30 & 16 \\
\hline
\end{tabular}

Note. The distribution of genotypes polymorphisms (Ins/Ins, Ins/Del, Del/Del) was determined in accordance with the law of genetic equilibrium Hardy-Weinberg; significant differences were defined by criterion $\chi^{2}-$ adjustment for Yates continuity.

Table 2. Systematics of generalized periodontitis by 'immunohistochemical' and 'genetic' profile of the individual

\begin{tabular}{|c|c|c|}
\hline T lymphocytes (CD-4) & T lymphocytes (CD-3) & B lymphocytes (CD-20) \\
\hline Genotype (I/I) & Genotype (D/I) & Genotype (D/D) \\
\hline Stable generalized periodontitis & $\begin{array}{c}\text { Conditionally stable generalized } \\
\text { periodontitis }\end{array}$ & $\begin{array}{c}\text { Progressive generalized peri- } \\
\text { odontitis }\end{array}$ \\
\hline
\end{tabular}

\section{Conclusions}

The results of correlation analysis indicate that genotype (Del/Del), as polymorphic variant of gene transcription factor NF-kB1, was significantly associated with the emergence of rapidly progressive periodontitis in young people.

Thus, nowadays, there is need to consider in the diagnosis of generalized periodontitis a polymorphic variants of genes, i.e. polymorphism transcription factor NF-kB1, which can enable detailing pathogenetic mechanisms of periodontitis in order to predict the emergence and development of nosology units and might be a genetic background for the justification and development of prevention and treatment measures. 


\section{References}

1. Bourgeois D. Epidemiology of periodontal status in dentate adults in France.J Periodontal Res. 2007;42(3):219-227.

2. Hasiuk NV. Morphofunctional organization gum in normal and inflammation. Simferopol: S. Heorhiyevsky Crimea State Medical University; 2009.

3. Hasiuk NV. Description of polymorphic variants of nuclear transcription factor NF-kB1 as predictors of generalized periodontitis development. Ukrainian Scientific Medical Youth Journal. 2016; 1(93):105-107.

4. Hasiuk NV. Cytological and cytogenetic features of the oral mucosa in human normal and inflammation. Kyiv: A. Bohomolets National Medical University; 2015.

5. Hrudyanov AI. Periodontal Diseases. Moscow: Medical News Agency; 2009:336.

6. Kornman KS. The innovation dilemma for periodontists. J Periodontol. 2010;81(5):646-649.

7. Kononen E. Population-based study of salivary carriage of periodontal pathogens in adults. J Clin Microbiol. 2007;45(8):2446-2451.

8. Rebrova OYu. Statistical analysis of medical data. Application of the application software STATISTIKA. Moscow: Media Field; 2002:312.
9. Schmalz G. Release of prostaglandin E2, IL-6 and IL-8 from human oral epithelial culture models after exposure to compounds of dental materials. Eur J Oral Sci. 2010;108:442-448.

10. Schnare M. Toll-like receptors: sentinels of host defense against bacterial infection. J Allergy Immunol. 2006;(139):75-85.

11. Shi D. Inflammatory bowel disease requires the interplay between innate and adaptive immune signals. Cell Res. 2006;(16):70-74.

12. Schulz S. Single nucleotide polymorphisms in interleukin-1gene cluster and subgingival colonization with Aggregatibacter actinomycetemcomitans in patients with aggressive periodontitis. Hum Immunol. 2011;72(10):940-946.

13. Schulz $\mathrm{S}$. The del/del genotype of the nuclear factor-kappa B-94ATTG polymorphism and its relation to aggressive periodontitis, et al. J Periodontal Res. 2010;45(3):396-403.

14. Van Dyke TE. Inflammation and factors that may regulate inflammatory response. J Periodontol. 2008;79(8):1503 -1507. 